\title{
Pattern formation in finite size non-equilibrium systems and models of morphogenesis
}

\author{
Yih-Yuh Chent and M C Crossł \\ $\dagger$ Physics Department, National Taiwan University, Taipei, Taiwan, Republic of China \\ $\$$ Condensed Matter Physics 114-36, California Institute of Technology, Pasadena, CA 91125 , \\ USA
}

Received 23 September 1993

Recommended by D I Meiron

\begin{abstract}
Two canonical pattern forming systems, the Rayleigh-Benard convection and the Turing mechanism for biological pattern formation, are compared. The similarity and fundamental differences in the mathematical structure of the two systems are addressed, with special emphasis on how the linear onset of patterns is affected by the finite size and the boundary conditions. Our analysis is facilitated by continuously varying the boundary condition, from one that admits simple algebraic solution of the problem but is unrealistic to another which is physically realizable. Our investigation shows that the size dependence of the convection problem can be considered generic, in the sense that for the majority of boundary conditions the same trend is to be observed, while for the corresponding Turing mechanism one will rely crucially on the assumed boundary conditions to ensure that a particular sequence of patterns be picked up as the system grows in size. This suggests that, although different systems might exhibit similar pattem forming features, it is still possible to distinguish them by characteristics which are specific to the individual models.
\end{abstract}

PACS numbers: $4720,4725,8710$

\section{Introduction}

Turing [1] suggested that instabilities at non-zero wavenumber in systems of reacting and diffusing chemicals could underlie morphogenesis in developing organisms. There has been much work developing this idea. On the other hand, instabilities at non-zero wavenumber are widely studied in many fluid systems, such as Rayleigh-Bénard convection, where thermally induced buoyancy forces drive a pattern of convection rolls between horizontal plates of a good thermal conductor.

At the simplest level of a description in an ideal infinite system the analogies between these two classes of systems are very strong. The similarities are brought out by a linear stability analysis of the spatially uniform state, seeking (from the translational invariance) a growing Fourier mode at a wavevector $q$ with growth rate $\lambda(q)$, i.e. a time dependence $\exp [\lambda(q) t]$. The passage through the imaginary axis of the maximum growth rate $\lambda\left(q_{\mathrm{c}}\right)$ for 'critical wavevector' $q_{\mathrm{c}}$ as a control parameter (which we will call $R$ ) is varied signals the development of a spatial structure with length scale $l_{c}=2 \pi q_{c}^{-1}$. This structure may be singly periodic (as in the rolls of Rayleigh-Benard convection) or a square or rectangular lattice (as in convection between poor conductors), but in a rotationally symmetric system is generically to a hexagonal cellular lattice [2]. The competition between these states, which 
can be constructed from the growth of a superposition of modes with wavenumber $q_{\mathrm{c}}$ but different orientations of the wavevectors, requires a nonlinear analysis [2].

Many common features in diverse pattern forming systems can be understood in terms of this linear stability analysis and the weakly nonlinear theory based on the nonlinear interaction of a band of unstable modes around $q_{\mathrm{c}}$ as described by 'amplitude' or 'envelope' equations [3]. Crudely, many, physically quite different, systems behave in the same way since the behaviour is given by the interaction of a few unstable eigenvectors: the different physical properties are all hidden in a small number of interaction parameters that control the behaviour.

One unfortunate consequence of this 'universality' of behaviour is that resulting patterns in a model are insensitive to the structure of the model, so that reproducing observed results is rarely a good test of the particular modelling. (We use the term 'universality' only in a qualitative sense, not in the much stronger quantitative sense that arises in discussions of second order phase transitions.) Simply from the agreement between predicted and observed macroscopic patterns, little is learned even about the correctness of the broad class of underlying microscopic mechanism (e.g. reaction and diffusion or more complicated models involving, in addition, flow or stresses and strains), let alone details of the parameters.

Particularly in biological systems the small finite size of the system is important: in many examples only a few basic periods $l_{\mathrm{c}}$ fit into the system. This has been exploited in some models. For example, the different characteristic patterns depending on the size of the system compared with the wavelength $l_{\mathrm{c}}$ at the time the spatial structure develops has been used by Murray [4] to explain the diversity of animal coat markings. On the other hand, the general insensitivity in other situations of morphogenesis to the actual size of the embryo has been used to argue against the Turing mechanism [5].

In this paper we discuss the role of boundary conditions in finite size pattern forming systems, in particular comparing and contrasting two canonical examples-RayleighBénard convection and reacting and diffusing chemicals-as well as giving a more general mathematical characterization. A major motivation of this work is to investigate the specificity of particular classes of models to observed phenomena that we have seen is not present in the ideal infinite system. We will concentrate entirely on the linear instability, in particular asking how the critical control parameter $R_{\mathrm{c}}$ for the onset of the instability to a state with spatial structure depends on the size of the system (characterized by a dimension $L$ ) and the form of the boundary conditions. We will find that the results on this very simple questions differ enormously for our two canonical systems: it is this difference that we want to explore and explain. A major result of this analysis is that the successful modelling of some morphogenetic phenomena seems quite specific to reaction-diffusion systems with sensible (no flux) boundary conditions: other physical mechanisms with their natural boundary conditions would not lead to the same phenomena.

To motivate the interest in the question of $R_{\mathrm{c}}(L)$ we first describe a particular, rather well documented, example of modelling morphogenesis: this is the example of the imaginal disk of the fly's wing developed by Kauffman et al [6-8]. We then describe the physical formulation of our canonical examples, and a mathematical characterization of the important differences in their structure. We next describe specific results that can be derived for these two systems, before presenting a general analysis of various types of behaviour.

\section{The fly wing model}

Kauffman et al [6] have proposed a Turing type model to explain the diverse experimental characteristics observed in a developing fly egg, such as transdetermination. This describes 
the phenomenon that even before metamorphosis begins inside a larva, the features typical of a particular adult appendage can be artificially induced by injection of the culture of the imaginal disc associated with that appendage. The model aims at reproducing the compartmental boundaries that appear during the early stage of egg development and later in the imaginal discs which eventually evolve-into different adult parts, and explaining the functional commitment of each zone separated by them in one unified mechanism. In their linear model, at least two chemicals are introduced so that their diffusion and mutual interaction in the isolated developing embryo, which admits no exchange of the chemicals across its boundary and is assumed to grow in a geometrically similar way, will generate spatial patterns that can become unstable only when its size reaches some specific values, say $L_{1}, L_{2}, \ldots$. The nodal lines of at least one of these chemicals are then identified with the compartmental lines on the embryo. Because the spatial patterns picked up by the system appear sequentially, a binary code characterizing each partition defined by the compartmental lines that are laid down at different times becomes possible. This binary address not only serves as a spatial information code but is also hypothesized to carry the concept of morphogenetic closeness of each disc, e.g. the antenna is closer to the genital than it is to the wing. The mathematical problem associated with this type of pattern formation models can be formulated quite generally in the following way:

$$
\lambda \psi=\hat{D} \nabla^{2} \psi+\hat{A} \psi \quad \text { in } \Omega
$$

where $\psi$ is an $m \times 1$ column vector representing the concentration of the $m$ diffusing and reacting chemicals (called morphogenes) inside the domain $\Omega$ with boundary $\partial \Omega, \hat{D}$ is a diagonal matrix consisting of their associated diffusion constants, and $\hat{A}$ is an $m \times m$ matrix signifying the linearized interactions among the morphogens. (We choose variables so that the uniform state is described by $\psi=0$.) For reacting chemicals in an isolated system we impose the no-flux boundary condition:

$$
\frac{\partial \psi}{\partial n}=0 \quad \text { on } \partial \Omega
$$

and the matrices $\hat{D}$ and $\hat{A}$ must be assumed to have been fine-tuned in such a way that the real part of the growth rate $\lambda$ is less than zero unless the system size $L$ is 'just right' ( $L=L_{1}$ or $\left.L_{2}, \ldots\right)$ to make it vanish. The different zones separated by the nodal lines of the eigenfunction $\psi$ associated with this marginally stable state then constitute the spatial pattern defined by the compartmental lines. To be specific, we can solve (1) and (2) by taking $\psi \equiv \varphi \bar{c}$, where $\bar{c} \equiv$ a constant $m \times 1$ vector to be determined, and $\varphi$ is an eigenfunction of the Laplacian operator subject to the Neumann boundary condition:

$$
\begin{aligned}
& -\nabla^{2} \varphi \equiv \mu \varphi \\
& \frac{\partial \varphi}{\partial n}=0 \quad \text { on } \partial \Omega
\end{aligned}
$$

for some eigenvalue $\mu$. (Notice that the boundary condition (2) is indeed consistent with the assumption that all components of the vector $\psi$ have the same spatial dependence given by $\varphi$.) Substituting this ansatz into (1) and requiring that a nontrivial solution exists, we obtain

$$
\operatorname{det}(-\lambda-\mu \hat{D}+\hat{A})=0
$$

which can be inverted to yield $\lambda=\lambda(\mu)$. If the parameters of the problem are suitably adjusted, then $\lambda(\mu)$ has a negative real part unless $\mu$ equals some critical value $\mu_{\mathrm{c}}$, in which case the system assumes marginal stability: $\operatorname{Re}(\lambda)=0$. Because the Laplacian eigenvalue $\mu$ is of the form $\mu=q_{j}^{2} / L^{2}$ ( $q_{j}=$ constants indexed by $j=1,2, \ldots$ ), we immediately conclude that 
the system can become marginally stable only if the dimension $L$ equals some $L_{j} \equiv q_{j} / \sqrt{\mu_{\mathrm{c}}}$. Another way of putting it is to say that the system always picks up a solution which has the intrinsic wavenumber $q_{\mathrm{c}}=\sqrt{\mu_{\mathrm{c}}}$. The important ingredient of this model is that as the system grows $\operatorname{Re}(\lambda)$ will successively return to the same value zero by constantly switching to a different mode, as shown in figure 1 .

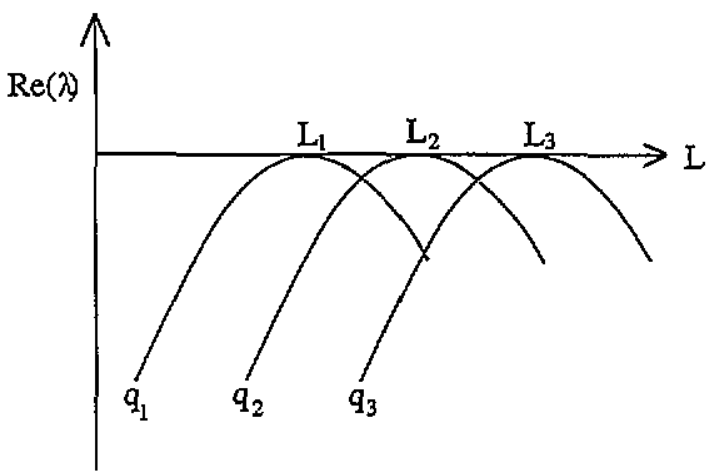

Figure 1. Different modes with wavenumbers $q_{1}, q_{2}, q_{3}, \ldots$ of the Turing model return to the same marginal statibility (growth rate $\operatorname{Re}(\lambda)=$ 0 ) at different sizes.

Exactly the same mechanism has also been proposed by Murray [4] to explain the animal coat markings. This time, a specific 'prepattern' is assumed to be acquired at a particular stage of the embryogenesis when a particular eigenmode is selected. Depending on the geometry and the size of the specific region under consideration, the patterns generated by this model bear features that seem to agree with what are seen in real animals, though firm experimental verification is still unavailable, and, as remarked in the introduction, mere resemblance of patterns should not be used alone as a reliable criterion for the validity of a theory.

Interestingly enough, another firmly established pattern forming system in fiuid mechanics, Rayleigh-Bénard convection, can also be formulated in such a way that its governing equations look very much like (1). To bring out the similarity and differences of these two systems, we will first give a brief description of the convection problem in the next section, then examine their mathematical structures explicitly.

\section{Rayleigh-Bénard convection}

An arbitrarily shaped shallow box of fluid remains quiescent unless the negative vertical temperature gradient applied to the two horizontal bounding surfaces exceeds a certain critical value, in which case a series of counterrotating convection rolls will form spontaneously (figure 2). The width of the rolls is observed to be always about the same as the depth of the fluid layer. In other words, the system picks up a particular wavelength at onset. Let $u, \theta$ and $p$ stand for the deviation of fluid velocity, temperature and pressure from the static pure conduction state, respectively. Under suitable normalization, the growth rate $\lambda$ and its associated eigenmode of the system obey the following equation [9]

$$
\begin{gathered}
\lambda \psi=\left\{\left(\begin{array}{cccc}
\sigma & 0 & 0 & 0 \\
0 & \sigma & 0 & 0 \\
0 & 0 & \sigma & 0 \\
0 & 0 & 0 & 1
\end{array}\right) \nabla^{2}+\left(\begin{array}{cccc}
0 & 0 & 0 & 0 \\
0 & 0 & 0 & 0 \\
0 & 0 & 0 & \sqrt{\sigma R} \\
0 & 0 & \sqrt{\sigma R} & 0
\end{array}\right)\right\} \psi-\left(\begin{array}{c}
\partial p / \partial x \\
\partial p / \partial y \\
\partial p / \partial z \\
0
\end{array}\right) \\
\equiv\left\{\hat{D} \nabla^{2}+\hat{A}\right\} \psi-\left(\begin{array}{c}
\nabla p \\
0
\end{array}\right)
\end{gathered}
$$


where $\hat{D}$ and $\hat{A}$ are $4 \times 4$ matrices defined in an obvious way, and

$$
\psi \equiv\left(\begin{array}{l}
u \\
\theta
\end{array}\right)
$$

is an abstract eigenfunction to the system. In the above, $R$, the Rayleigh number, is the temperature difference established across the fluid layer and is a convenient control parameter for the system, while $\sigma$, the Prandtl number, measures the viscosity in units of fluid's thermal diffusivity. The equation which involves $\nabla^{2} u$ is the Navier-Stokes equation with the buoyancy term $(\alpha \theta)$ included, whereas the $\nabla^{2} \theta$ equation describes the heat transport in the fluid. In addition, the fluid also has to satisfy the incompressibility condition $\nabla \cdot u=0$.

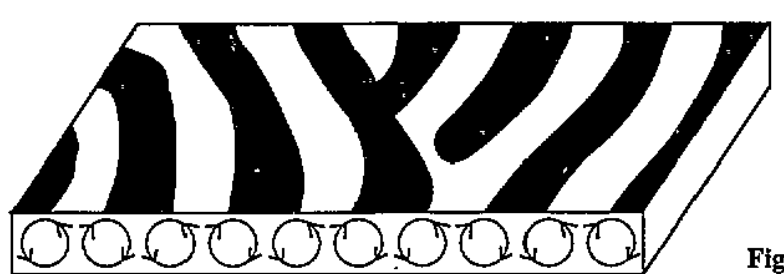

Figure 2. Counterrotating rolls appear at convective onset. The shadowgraphic image of the convection pattern is schematically shown in interlaced bright and dark bands.

Since (1) is very much like (4) in appearance, one might hope that, under suitable modifications, the trick we used to solve for the former will work equally well for the latter. To see how this is achieved we can take a Neumann eigenfunction $\varphi$ of the two dimensional Laplacian $-\nabla_{h}^{2}$, which is defined on any horizontal cross-section of the box, and assume $\psi=\left(\bar{u} \frac{\partial \varphi}{\partial x}, \bar{u} \frac{\partial \varphi}{\partial y}, \bar{u}_{z} \varphi, \bar{\theta} \varphi\right)^{T}$, where $\bar{u}, \bar{u}_{z}$ and $\bar{\theta}$ are functions of the vertical coordinate $z$ alone that must be determined by the boundary conditions on the two horizontal walls. Interpreting $\mu$ as the eigenvalue associated with $\varphi$, we can derive a determinantal equation similar to (3) and write down a 'dispersion relation' $\lambda=\lambda(\mu)$. Now we can copy what we did in the previous section: if the parameter $R$ is suitably adjusted then $\lambda$, which can be shown to be real-valued for this problem because the matrix $\hat{A}$ is Hermitian, will always be less than zero unless $\mu=\mu_{\mathrm{c}}$, at which value the system acquires marginal stability. What this implies is that the $\lambda$ versus $L$ (= lateral dimension) plot for this system must look just like figure 1 . The physics underlying the constant switching of unstable modes shown in this figure is simply this: the system prefers to accommodate an integral number of convection rolls of the same width and, as a result of our enlarging the system size $L$, it always switches to a different state with one more roll whenever enough room is created to make this possible.

Unfortunately there is a serious catch in this method, namely, the lateral boundary conditions intrinsic to this simple ansatz are not realizable in experiments. Specifically, in order for the Neumann boundary conditions on $\varphi$ to be valid we have to assume that (1) the sidewall is a perfect insulator, (2) there is a 'stress free' condition on the vertical velocity and (3) the fluid does not exhibit any local rotational motion about the $z$-axis near the sidewall [9]. A thermally insulated sidewall certainly can be arranged in a controlled experiment-although there is also interest in studying how other thermal properties will affect the stability - but the same is not true for the fluid's viscous boundary condition. In general, the most natural 'no-slip' condition, i.e. $u=0$ at the sidewall, is incompatible with 
the artificial 'stress-free' and 'rotation-free' conditions. The physically natural boundary conditions are not consistent with the ansatz of like spatial variation for all the different variables.

To remove the limitation on allowed thermal sidewalls and overcome the difficulties associated with the viscous boundary condition while retaining the attractive features of this simple method, we can perturb the boundary conditions from this known solution gradually until we finally teach the physically realizable one. This trick of 'path-following' is commonly used in numerical work to solve for the nontrivial solution to an equation when only a particular (and usually trivial) solution is known. What is interesting is that this same trick can be applied here analytically, due to the fact that a variational formulation for the current problem is possible $[9,10]$. The conclusions we arrive at from this approach are two-fold. On the one hand, we can show that, as expected, quantitatively the stability curve $\lambda(L)$ is insensitive to the physical boundary conditions imposed at the sidewall, i.e. the fractional changes are small, when $L$ is large. But on the other hand, the qualitative feature of it changes dramatically as soon as one turns on the perturbation. In fact, we can show that for all boundary conditions other than the one we start with, $\lambda(L)$ is given to leading order by $-a L^{-2}$ for some positive constant $a$ which is independent of the sidewall boundary condition, provided $L$ is large enough. (Here 'large' is measured with respect to a cross-over size which is of order the characteristic wavelength but is sensitive to what boundary condition is imposed when the perturbation is very small [9].) This phenomenon is shown schematically in figure 3 . These results can also be easily confirmed in a one dimensional situation using the amplitude equation mentioned earlier $[11,12]$.

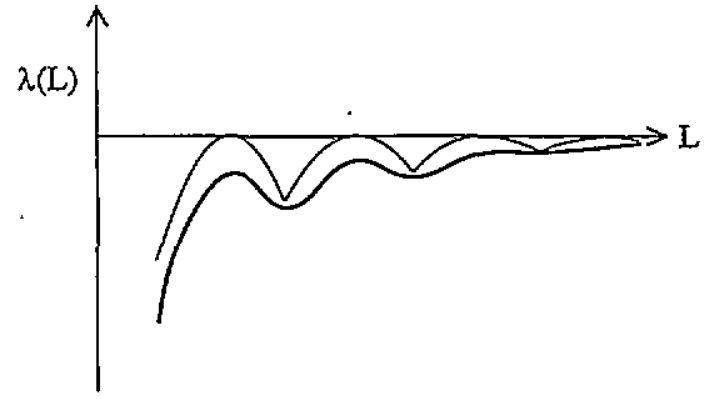

Figure 3. The oscillatory stability curve (light) associated with the unrealistic boundary conditions which admit simple algebraic solution for the convection problem immediately acquires the $L^{-2}$ trend upon perturbation (dark).

Using this approach we can also derive a number of results of interest within the convection problem. We can show that the sidewall always has a stabilizing effect on the system, though its effect usually is partially masked by another competing factor coming from the system's preference of accommodating just the right number of convection rolls, which was discussed before. It is the combination of these two factors which results in the superposition of oscillation on the monotonic background in figure 3. (However, for a rigid and perfectly conducting sidewall we can prove that the dependence is monotonic.) In addition, the sidewall thermal conductivity affects the stability in a monotonic way: the larger the conductivity, the higher the stability. Another merit of this approach is that we can actually obtain bounds on either the growth rate or the critical Rayleigh number $R_{\mathrm{c}}$. For instance, we can show that the most unstable mode of a rigid cell is still more stable than the second unstable mode of a cell whose side is rotation-free. 
As a side remark, we would like to point out that, although it is a common practice in the literature to replace the sidewall by a simplified thermal boundary condition which involves only a suitable linear combination of $\theta$ and $\frac{\partial \theta}{\partial n}$, and although our previous analysis can be most easily done when this simplification is made, our conclusions nevertheless are valid for a convection cell with a real sidewall which can have arbitrary geometry and thickness. The details of this aspect are reported elsewhere [10].

\section{Comparison of the two systems}

The major conclusion we wish to derive from sections 2 and 3 is that for the reactiondiffusion system the physically natural boundary conditions lead to a $\lambda(L)$ that periodically returns to a fixed maximum value as $L$ increases to infinity. Thus a system tuned to be near this maximum threshold value at small sizes will return to threshold, with the onset of different modes, as $L$ increases, whilst remaining below threshold for intermediate values of $L$. On the other hand, for the convection problem, the natural boundary conditions lead to a $\lambda(L)$ (or equivalently, $R_{\mathrm{c}}(L)$ ) which has a systematic trend (as well as a superimposed oscillatory part) as $L$ changes: in general, tuning $R$ to threshold at small system sizes will lead to a strongly nonlinear situation if $L$ is increased. This latter behaviour is typical of pattern forming equations with an arbitrary choice of boundary conditions, and is particularly clear in a convection cell with a rigid, perfectly conducting side. Only for carefully tuned boundary conditions will the behaviour of figure 1 be recovered. To summarize: the most natural boundary conditions for the convection problem can be considered generic in the sense that they belong to a much larger class of mathematical constraints which all produce the same qualitative features; and the opposite is true for the Turing mechanism.

Another difference occurs when the variables of both systems are ali clamped at the boundary. While for the reaction-diffusion system we will get the same dispersion relation (equation (3)), and hence the same feature displayed in figure 1, the opposite is true for the convection system: we can show that the growth rate is a strictly increasing function of system size $L$.

It is amusing to note that the Hermitian property of the convection problem that simplifies many of the proofs must not apply in the reaction-diffusion systems if a single finite wavelength is to be selected. As a matter of fact, we can show that the growth rate of a reaction-diffusion system with a Hermitian $\hat{A}$ must always increase monotonically when the system is stretched in the lateral directions [9]. (Incidentally, one must not confuse this with the well-known fact that the lowest excitation energy for a smaller vibrating membrane is greater than that for a larger one when their circumferences are clamped! The major difference lies, of course, in the boundary conditions imposed-one is Dirichlet, and the other is Neumann.) This means the system is either always stable, or wavenumbers ranging from zero to a particular value will be excited altogether, with the uniform state having the largest growth rate. Clearly, this result does not apply to the convection system because it has to satisfy the additional incompressibility condition.

\section{Conclusion}

With the greatly increased interest in studying complex systems and the mushrooming of different pattern forming systems from very diverse fields during the past few decades, it is necessary to identify the elements in a model that are responsible for bringing out the observed similarity or difference in its exhibited patterns or behaviour compared with 
other models. In this work we have analysed two of the most commonly studied systems, the Rayleigh-Benard convection and the Turing mechanism of developmental biology, and clearly illustrated how the respective natural boundary conditions lead to different properties near onset of instability. While for the biological model it is natural to assume the no-flux boundary condition, and thus allowing a single Laplacian eigenfunction to carry the spatial variation of all the chemicals inside the biological system, the solution characteristic to this model nevertheless only represents a highly specialized case among the many other possibilities. As our analysis of the convection problem shows, for the majority of boundary conditions the finiteness of a system generally leads to a trend that is modified only by minor oscillations. This should be contrasted with the biological model in which the stability curve always returns to the same critical level when the system size increases. Our study therefore suggests that boundary conditions particular to a specific model sometimes do give rise to different characteristics that can distinguish that particular system from others.

\section{Acknowledgment}

One of us (MCC) thanks P C Hohenberg for many discussions on the importance of boundary effects on pattern formation in non-equilibrium systems. We thank the NSF for support under grant number DMR-9013984.

\section{References}

[1] Turing A M 1952 Phil. Trans. R. Soc. B 237 37-72

[2] Busse F H 1978 Rep. Prog. Phys. 41 1929-67

[3] Newcll A C 1974 Envelope equations Lectures in Applied Mechanics vol. 15 (Providence, RI: American Mathematical Society) pp 157-63

[4] Murray J D 1989 Mathematical Biology (Berlin: Springer) and references cited therein

[5] Pate E and Othmer H G 1984 Differentiation 28 1-8

[6] Kauffman S A, Shymko R and Trabert K 1978 Science 199 259-70

[7] Kauffman S A 1981 Phil Trans. R. Soc. B 295 567-94

[8] Bunow B, Kernevez J-P, Joy G and Thomas D 1980 J. Theor. Biol. $84629-49$

[9] Chen Yih-Yuh 1992 J. Fluid Mech. $241549-85$

[10] Chen Yih-Yuh 1992 Phys. Rev. A 45 3727-31

[11] Ahlers G, Cross M C, Hohenberg P C and Safran S 198t J. Fluid Mech. 110 297-334

[12] Cross M C, Daniels P G, Hohenberg P C and Siggia E D 1983 J. Fluid Mech. 127 155-83 